\title{
ENCAPSULATING MULTIPLE COMMUNICATION-COST METRICS IN PARTITIONING SPARSE RECTANGULAR MATRICES FOR PARALLEL MATRIX-VECTOR MULTIPLIES*
}

\author{
BORA UÇAR ${ }^{\dagger}$ AND CEVDET AYKANAT ${ }^{\dagger}$
}

\begin{abstract}
This paper addresses the problem of one-dimensional partitioning of structurally unsymmetric square and rectangular sparse matrices for parallel matrix-vector and matrix-transposevector multiplies. The objective is to minimize the communication cost while maintaining the balance on computational loads of processors. Most of the existing partitioning models consider only the total message volume hoping that minimizing this communication-cost metric is likely to reduce other metrics. However, the total message latency (start-up time) may be more important than the total message volume. Furthermore, the maximum message volume and latency handled by a single processor are also important metrics. We propose a two-phase approach that encapsulates all these four communication-cost metrics. The objective in the first phase is to minimize the total message volume while maintaining the computational-load balance. The objective in the second phase is to encapsulate the remaining three communication-cost metrics. We propose communicationhypergraph and partitioning models for the second phase. We then present several methods for partitioning communication hypergraphs. Experiments on a wide range of test matrices show that the proposed approach yields very effective partitioning results. A parallel implementation on a PC cluster verifies that the theoretical improvements shown by partitioning results hold in practice.
\end{abstract}

Key words. matrix partitioning, structurally unsymmetric matrix, rectangular matrix, iterative method, matrix-vector multiply, parallel computing, message volume, message latency, communication hypergraph, hypergraph partitioning

AMS subject classifications. 05C50, 05C65, 65F10, 65F50, 65Y05

DOI. $10.1137 / \mathrm{S} 1064827502410463$

1. Introduction. Repeated matrix-vector and matrix-transpose-vector multiplies that involve the same large, sparse, structurally unsymmetric square or rectangular matrix are the kernel operations in various iterative algorithms. For example, iterative methods such as the conjugate gradient normal equation error and residual methods (CGNE and CGNR) [15, 34] and the standard quasi-minimal residual method (QMR) [14], used for solving unsymmetric linear systems, require computations of the form $y=A x$ and $w=A^{T} z$ in each iteration, where $A$ is an unsymmetric square coefficient matrix. The least squares (LSQR) method [31], used for solving the least squares problem, and the Lanczos method [15], used for computing the singular value decomposition, require frequent computations of the form $y=A x$ and $w=A^{T} z$, where $A$ is a rectangular matrix. Iterative methods used in solving the normal equations that arise in interior point methods for linear programming require repeated computations of the form $y=A D^{2} A^{T} z$, where $A$ is a rectangular constraint matrix and $D$ is a diagonal matrix. Rather than forming the coefficient matrix $A D^{2} A^{T}$, which may be quite dense, the above computation is performed as $w=A^{T} z, x=D^{2} w$, and $y=A x$. The surrogate constraint method $[29,30,39,40]$, which is used for solving the linear feasibility problem, requires decoupled matrix-vector and matrix-transpose-vector multiplies involving the same rectangular matrix.

${ }^{*}$ Received by the editors July 1, 2002; accepted for publication (in revised form) October 13, 2003; published electronically May 25, 2004. This work was partially supported by The Scientific and Technical Research Council of Turkey (TÜBITAK) under grant $199 \mathrm{E} 013$.

http://www.siam.org/journals/sisc/25-6/41046.html

${ }^{\dagger}$ Computer Engineering Department, Bilkent University, Ankara, 06800, Turkey (ubora@cs. bilkent.edu.tr, aykanat@cs.bilkent.edu.tr). 
In the framework of this paper, we assume that no computational dependency exists between the input and output vectors $x$ and $y$ of the $y=A x$ multiply. The same assumption applies to the input and output vectors $z$ and $w$ of the $w=A^{T} z$ multiply. In some of the above applications, the input vector of the second multiply is obtained from the output vector of the first one - and vice versa - through linear vector operations because of intra- and interiteration dependencies. In other words, linear operations may occur only between the vectors that belong to the same space. In this setting, $w$ and $x$ are $x$-space vectors, whereas $z$ and $y$ are $y$-space vectors. These assumptions hold naturally in some of the above applications that involve a rectangular matrix. Since $x$ - and $y$-space vectors are of different dimensions, they cannot undergo linear vector operations. In the remaining applications, which involve a square matrix, a computational dependency does not exist between $x$-space and $y$-space vectors because of the nature of the underlying method. Our goal is the parallelization of the computations in the above iterative algorithms through rowwise or columnwise partitioning of matrix $A$ in such a way that the communication overhead is minimized and the computational-load balance is maintained.

In the framework of this paper, we do not address the efficient parallelization of matrix-vector multiplies involving more than one matrix with different sparsity patterns. Handling such cases requires simultaneous partitioning of the participating matrices in a method that considers the complicated interaction among the efficient parallelizations of the respective matrix-vector multiplies (see [21] for such a method). The most notable cases are the preconditioned iterative methods that use an explicit preconditioner such as an approximate inverse $[3,4,16] M \approx A^{-1}$. These methods involve matrix-vector multiplies with $M$ and $A$. The present work can be used in such cases by partitioning matrices independently. However, this approach would suffer from communication required for reordering the vector entries between the two matrix-vector multiplies.

The standard graph-partitioning model has been widely used for one-dimensional (1D) partitioning of square matrices. This approach models matrix-vector multiply $y=A x$ as a weighted undirected graph and partitions the vertices such that the parts are equally weighted and the total weight of the edges crossing between the parts is minimized. The partitioning constraint and objective correspond to, respectively, maintaining the computational-load balance and minimizing the total message volume. In recent works, Çatalyürek [6], Çatalyürek and Aykanat [7], and Hendrickson [19] mentioned the limitations of this standard approach. First, it tries to minimize a wrong objective function since the edge-cut metric does not model the actual communication volume. Second, it can only express square matrices and produce symmetric partitioning by enforcing identical partitions on the input and output vectors $x$ and $y$. Symmetric partitioning is desirable for parallel iterative solvers on symmetric matrices because it avoids the communication of vector entries during the linear vector operations between the $x$-space and $y$-space vectors. However, this symmetric partitioning is a limitation for iterative solvers on unsymmetric square or rectangular matrices when the $x$-space and $y$-space vectors do not undergo linear vector operations.

Recently, Aykanat, Pinar, and Çatalyürek [2], Çatalyürek and Aykanat [7, 8], and Pinar, Çatalyürek, Aykanat, and Pinar [32] proposed hypergraph models for partitioning unsymmetric square and rectangular matrices with the flexibility of producing unsymmetric partitions on the input and output vectors. Hendrickson and Kolda [21] proposed a bipartite graph model for partitioning rectangular matrices with the same flexibility. A distinct advantage of the hypergraph model over the bipartite graph model is that the hypergraph model correctly encodes the total message volume into 
its partitioning objective. Several recently proposed alternative partitioning models for parallel computing were discussed in the excellent survey by Hendrickson and Kolda [20]. As noted in the survey, most of the partitioning models mainly consider minimizing the total message volume. However, the communication overhead is a function of the message latency (start-up time) as well as the message volume. Depending on the machine architecture and problem size, the communication overhead due to the message latency may be much higher than the overhead due to the message volume [12]. None of the works listed in the survey address minimizing the total message latency. Furthermore, the maximum message volume and latency handled by a single processor are also crucial cost metrics to be considered in partitionings. As also noted in the survey [20], new approaches that encapsulate these four communicationcost metrics are needed.

In this work, we propose a two-phase approach for minimizing multiple communication-cost metrics. The objective in the first phase is to minimize the total message volume while maintaining the computational-load balance. This objective is achieved through partitioning matrix $A$ within the framework of the existing 1D matrix partitioning methods. The partitioning obtained in the first phase is an input to the second phase so that it determines the computational loads of processors while setting a lower bound on the total message volume. The objective in the second phase is to encapsulate the remaining three communication-cost metrics while trying to attain the total message volume bound as much as possible. The metrics minimized in the second phase are not simple functions of the cut edges or hyperedges or vertex weights defined in the existing graph and hypergraph models even in the multiobjective [37] and multiconstraint [25] frameworks. Besides, these metrics cannot be assessed before a partition is defined. Hence, we anticipate a two-phase approach. Pinar and Hendrickson [33] also adopt a multiphase approach for handling complex partitioning objectives. Here, we focus on the second phase and do not go back and forth between the phases. Therefore, our contribution can be seen as a postprocess to the existing partitioning methods. For the second phase, we propose a communication-hypergraph partitioning model. A hypergraph is a generalization of a graph in which hyperedges (nets) can connect more than two vertices. The vertices of the communication hypergraph, with proper weighting, represent primitive communication operations, and the nets represent processors. By partitioning the communication hypergraph into equally weighted parts such that nets are split among as few vertex parts as possible, the model maintains the balance on message-volume loads of processors and minimizes the total message count. The model also enables incorporating the minimization of the maximum message-count metric.

The organization of the paper is as follows. Section 2 gives background material on the parallel matrix-vector multiplies and hypergraph partitioning problem. The proposed communication-hypergraph and partitioning models are discussed in section 3. Section 4 presents three methods for partitioning communication hypergraphs. Finally, experimental results are presented and discussed in section 5 .

\section{Background.}

2.1. Parallel multiplies. Suppose that rows and columns of an $M \times N$ matrix $A$ are permuted into a $K \times K$ block structure

$$
A_{B L}=\left[\begin{array}{cccc}
A_{11} & A_{12} & \cdots & A_{1 K} \\
A_{21} & A_{22} & \cdots & A_{2 K} \\
\vdots & \vdots & \ddots & \vdots \\
A_{K 1} & A_{K 2} & \cdots & A_{K K}
\end{array}\right]
$$


for rowwise or columnwise partitioning, where $K$ is the number of processors. Block $A_{k \ell}$ is of size $M_{k} \times N_{\ell}$, where $\sum_{k} M_{k}=M$ and $\sum_{\ell} N_{\ell}=N$. In rowwise partitioning, each processor $P_{k}$ holds the $k$ th row stripe $\left[A_{k 1} \cdots A_{k K}\right]$ of size $M_{k} \times N$. In columnwise partitioning, $P_{k}$ holds the $k$ th column stripe $\left[A_{1 k}^{T} \cdots A_{K k}^{T}\right]^{T}$ of size $M \times N_{k}$. We assume that either row stripes or column stripes have a nearly equal number of nonzeros for having computational-load balance. We assume rowwise partitioning of $A$ throughout the following discussion.

2.1.1. Matrix-vector multiply. Consider an iterative algorithm involving repeated matrix-vector multiplies of the form $y=A x$, where $y$ and $x$ are column vectors of size $M$ and $N$, respectively. The rowwise partition of matrix $A$ defines a partition on the output vector $y$, whereas the input vector $x$ is partitioned conformably with the column permutation of $A$. That is, $y$ and $x$ vectors are partitioned as $y=\left[y_{1}^{T} \cdots y_{K}^{T}\right]^{T}$ and $x=\left[x_{1}^{T} \cdots x_{K}^{T}\right]^{T}$, where processor $P_{k}$ computes subvector $y_{k}$ of size $M_{k}$ while holding subvector $x_{k}$ of size $N_{k}$. In order to avoid communication during linear vector operations, all other $x$-space and $y$-space vectors are partitioned conformably with the partitions on $x$ and $y$ vectors, respectively.

Row-parallel $y=A x$ executes the following steps at each processor $P_{k}$ :

1. For each nonzero off-diagonal block $A_{\ell k}$, send sparse vector $\hat{x}_{k}^{\ell}$ to processor $P_{\ell}$, where $\hat{x}_{k}^{\ell}$ contains only those entries of $x_{k}$ corresponding to the nonzero columns in $A_{\ell k}$.

2. Compute the diagonal block product $y_{k}^{k}=A_{k k} \times x_{k}$, and set $y_{k}=y_{k}^{k}$.

3. For each nonzero off-diagonal block $A_{k \ell}$, receive $\hat{x}_{\ell}^{k}$ from processor $P_{\ell}$, then compute $y_{k}^{\ell}=A_{k \ell} \times \hat{x}_{\ell}^{k}$, and update $y_{k}=y_{k}+y_{k}^{\ell}$.

In step $1, P_{k}$ might be sending the same $x_{k}$-vector entry to different processors according to the sparsity pattern of the respective column of $A$. This multicast-like operation is referred to here as an expand operation.

2.1.2. Matrix-vector and matrix-transpose-vector multiplies. Consider an iterative algorithm involving repeated matrix-vector and matrix-transpose-vector multiplies of the form $y=A x$ and $w=A^{T} z$. A rowwise partition of $A$ induces a columnwise partition of $A^{T}$. Therefore, the partition on the $z$ vector defined by the columnwise partition of $A^{T}$ will be conformable with that on the $y$ vector. That is, $z=\left[z_{1}^{T} \cdots z_{K}^{T}\right]^{T}$ and $y=\left[y_{1}^{T} \cdots y_{K}^{T}\right]^{T}$, where $z_{k}$ and $y_{k}$ are both of size $M_{k}$. In a dual manner, the columnwise permutation of $A$ induces a rowwise permutation of $A^{T}$. Therefore, the partition on the $w$ vector induced by the rowwise permutation of $A^{T}$ will be conformable with that on the $x$ vector. That is, $w=\left[w_{1}^{T} \cdots w_{K}^{T}\right]^{T}$ and $x=\left[x_{1}^{T} \cdots x_{K}^{T}\right]^{T}$, where $w_{k}$ and $x_{k}$ are both of size $N_{k}$.

We use a column-parallel algorithm for $w=A^{T} z$ and the row-parallel algorithm for $y=A x$ and thus obtain a row-column-parallel algorithm. In $y=A x$, processor $P_{k}$ holds $x_{k}$ and computes $y_{k}$. In $w=A^{T} z, P_{k}$ holds $z_{k}$ and computes $w_{k}$.

Column-parallel $w=A^{T} z$ executes the following steps at processor $P_{k}$ :

1. For each nonzero off-diagonal block $\left(A^{T}\right)_{\ell k}=\left(A_{k \ell}\right)^{T}$, form sparse vector $\hat{w}_{\ell}^{k}$ which contains only those results of $w_{\ell}^{k}=\left(A^{T}\right)_{\ell k} \times z_{k}$ corresponding to the nonzero rows in $\left(A^{T}\right)_{\ell k}$. Send $\hat{w}_{\ell}^{k}$ to processor $P_{\ell}$.

2. Compute the diagonal block product $w_{k}^{k}=\left(A^{T}\right)_{k k} \times z_{k}$, and set $w_{k}=w_{k}^{k}$.

3 . For each nonzero off-diagonal block $\left(A^{T}\right)_{k \ell}$, receive partial-result vector $\hat{w}_{k}^{\ell}$ from processor $P_{\ell}$, and update $w_{k}=w_{k}+\hat{w}_{k}^{\ell}$.

The multinode accumulation on $w_{k}$-vector entries is referred to here as the fold operation. 


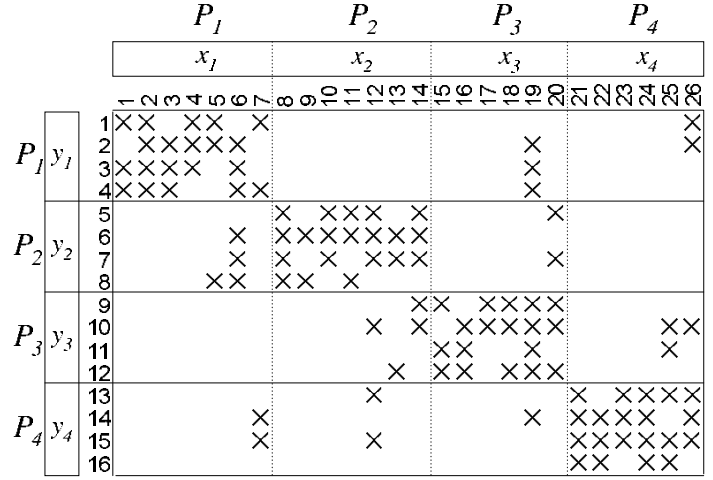

(a)

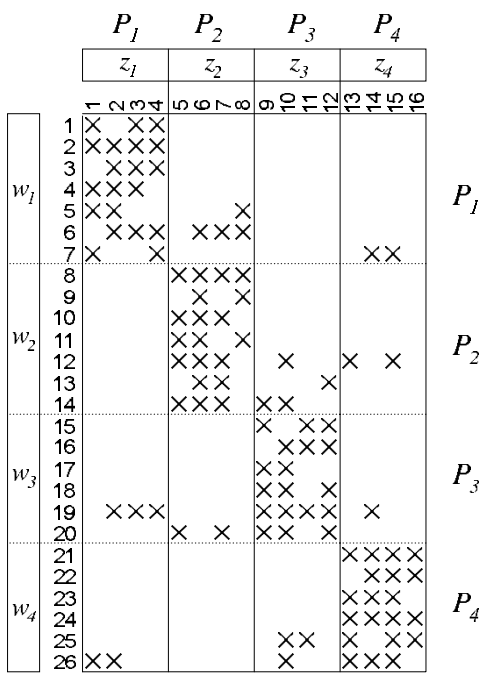

(b)

FIG. 2.1. $4 \times 4$ block structures of a sample matrix $A$ : (a) $A_{B L}$ for row-parallel $y=A x$ and (b) $\left(A^{T}\right)_{B L}$ for column-parallel $w=A^{T} z$.

2.1.3. Analysis. Here, we restate and summarize the facts given in $[7,21]$ for the communication requirement in the row-parallel $y=A x$ and column-parallel $w=A^{T} z$. We will use Figure 2.1 for a better understanding of these facts. Figure 2.1 displays $4 \times 4$ block structures of a $16 \times 26$ sample matrix $A$ and its transpose. In Figure 2.1(a), horizontal solid lines identify a partition on the rows of $A$ and on vector $y$, whereas vertical dashed lines identify virtual column stripes inducing a partition on vector $x$. In Figure 2.1(b), vertical solid lines identify a partition on the columns of $A^{T}$ and on vector $z$, whereas horizontal dashed lines identify virtual row stripes inducing a partition on vector $w$. The computational-load balance is maintained by assigning $25,26,25$, and 25 nonzeros to processors $P_{1}, P_{2}, P_{3}$, and $P_{4}$, respectively.

FACT 1. The number of messages sent by processor $P_{k}$ in row-parallel $y=A x$ is equal to the number of nonzero off-diagonal blocks in the kth virtual column stripe of $A$. The volume of messages sent by $P_{k}$ is equal to the sum of the number of nonzero columns in each off-diagonal block in the kth virtual column stripe.

In Figure 2.1(a), $P_{2}$, holding $x$-vector block $x_{2}=x[8: 14]$, sends vector $\hat{x}_{2}^{3}=x[12$ : 14] to $P_{3}$ because of nonzero columns 12,13 , and 14 in $A_{32} . P_{3}$ needs those entries to compute $y[9], y[10]$, and $y[12]$. Similarly, $P_{2}$ sends $\hat{x}_{2}^{4}=x[12]$ to $P_{4}$ because of the nonzero column 12 in $A_{42}$. Hence, the number of messages sent by $P_{2}$ is 2 with a total volume of four words. Note that $P_{2}$ effectively expands $x[12]$ to $P_{3}$ and $P_{4}$.

FACT 2. The number of messages sent by processor $P_{k}$ in column-parallel $w=A^{T} z$ is equal to the number of nonzero off-diagonal blocks in the kth column stripe of $A^{T}$. The volume of messages sent by $P_{k}$ is equal to the sum of the number of nonzero rows in each off-diagonal block in the kth column stripe of $A^{T}$.

In Figure 2.1(b), $P_{3}$, holding $z$-vector block $z_{3}=z[9: 12]$, computes the offdiagonal block products $w_{2}^{3}=\left(A^{T}\right)_{23} \times z_{3}$ and $w_{4}^{3}=\left(A^{T}\right)_{43} \times z_{3}$. It then forms vectors $\hat{w}_{2}^{3}$ and $\hat{w}_{4}^{3}$ to be sent to $P_{2}$ and $P_{4}$, respectively. $\hat{w}_{2}^{3}$ contains its contribution to $w[12: 14]$ due to the nonzero rows 12,13 , and 14 in $\left(A^{T}\right)_{23}$. Accordingly, $\hat{w}_{4}^{3}$ contains its contribution to $w[25: 26]$ due to the nonzero rows 25 and 26 in $\left(A^{T}\right)_{43}$. Hence, $P_{3}$ sends two messages with a total volume of five words. 
FACT 3. Communication patterns of $y=A x$ and $w=A^{T} z$ multiplies are duals of each other. If a processor $P_{k}$ sends a message to $P_{\ell}$ containing some $x_{k}$ entries in $y=$ $A x$, then $P_{\ell}$ sends a message to $P_{k}$ containing its contributions to the corresponding $w_{k}$ entries in $w=A^{T} z$.

Consider the communication between processors $P_{2}$ and $P_{3}$. In $y=A x, P_{2}$ sends a message to $P_{3}$ containing $x[12: 14]$, whereas in $w=A^{T} z, P_{3}$ sends a dual message to $P_{2}$ containing its contributions to $w[12: 14]$.

FACT 4. The total number of messages in the $y=A x$ or $w=A^{T} z$ multiply is equal to the number of nonzero off-diagonal blocks in $A$ or $A^{T}$. The total volume of messages is equal to the sum of the number of nonzero columns or rows in each off-diagonal block in $A$ or $A^{T}$, respectively.

In Figure 2.1, there are nine nonzero off-diagonal blocks, containing a total of 13 nonzero columns or rows in $A$ or $A^{T}$. Hence, the total number of messages in $y=A x$ or $w=A^{T} z$ is nine, and the total volume of messages is 13 words.

2.2. Hypergraph partitioning. A hypergraph $\mathcal{H}=(\mathcal{V}, \mathcal{N})$ is defined as a set of vertices $\mathcal{V}$ and a set of nets $\mathcal{N}$. Every net $n_{i}$ is a subset of vertices. The vertices of a net are also called its pins. The size of a net $n_{i}$ is equal to the number of its pins, i.e., $\left|n_{i}\right|$. The set of nets that contain vertex $v_{j}$ is denoted as $N e t s\left(v_{j}\right)$, which is also extended to a set of vertices appropriately. The degree of a vertex $v_{j}$ is denoted by $d_{j}=\left|N e t s\left(v_{j}\right)\right|$. Weights can be associated with vertices.

$\Pi=\left\{\mathcal{V}_{1}, \ldots, \mathcal{V}_{K}\right\}$ is a $K$-way vertex partition of $\mathcal{H}=(\mathcal{V}, \mathcal{N})$ if each part $\mathcal{V}_{k}$ is nonempty, parts are pairwise disjoint, and the union of parts gives $\mathcal{V}$. In $\Pi$, a net is said to connect a part if it has at least one pin in that part. The connectivity set $\Lambda_{i}$ of a net $n_{i}$ is the set of parts connected by $n_{i}$. The connectivity $\lambda_{i}=\left|\Lambda_{i}\right|$ of a net $n_{i}$ is the number of parts connected by $n_{i}$. In $\Pi$, weight of a part is the sum of the weights of vertices in that part.

In the hypergraph partitioning problem, the objective is to minimize the cutsize:

$$
\operatorname{cutsize}(\Pi)=\sum_{n_{i} \in \mathcal{N}}\left(\lambda_{i}-1\right)
$$

This objective function is widely used in the VLSI community, and it is referred to as the connectivity-1 metric [28]. The partitioning constraint is to maintain a balance on part weights, i.e.,

$$
\frac{W_{\max }-W_{a v g}}{W_{\text {avg }}} \leq \epsilon
$$

where $W_{\max }$ is the weight of the part with the maximum weight, $W_{\text {avg }}$ is the average part weight, and $\epsilon$ is a predetermined imbalance ratio. This problem is NP-hard [28].

3. Models for minimizing communication cost. In this section, we present our hypergraph partitioning models for the second phase of the proposed two-phase approach. We assume that a $K$-way rowwise partition of matrix $A$ is obtained in the first phase with the objective of minimizing the total message volume while maintaining the computational-load balance.

3.1. Row-parallel $\boldsymbol{y}=\boldsymbol{A x}$. Let $A_{B L}$ denote a block-structured form (see (2.1)) of $A$ for the given rowwise partition. 


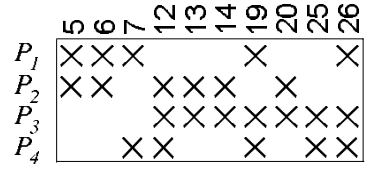

(a)

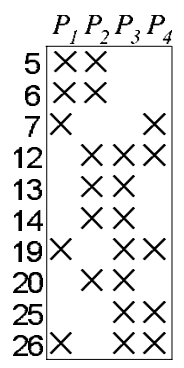

(b)

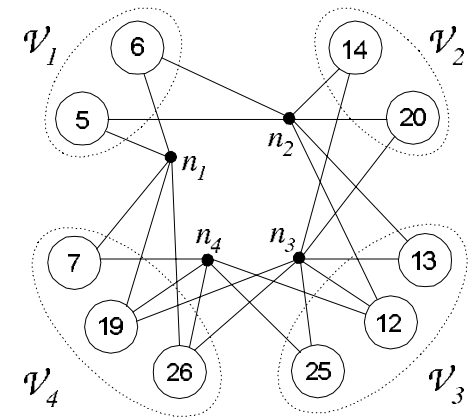

(c)

FIG. 3.1. Communication matrices (a) $C$ for row-parallel $y=A x$, (b) $C^{T}$ for column-parallel $w=A^{T} z$, and (c) the associated communication hypergraph and its four-way partition.

3.1.1. Communication-hypergraph model. We identify two sets of columns in $A_{B L}$ : internal and coupling. Internal columns have nonzeros only in one row stripe. The $x$-vector entries that are associated with these columns should be assigned to the respective processors to avoid unnecessary communication. Coupling columns have nonzeros in more than one row stripe. The $x$-vector entries associated with the coupling columns, referred to as $x_{C}$, necessitate communication. The proposed approach considers partitioning these $x_{C}$-vector entries to reduce the total message count and the maximum message volume. Consequences of this partitioning on the total message volume will be addressed in section 3.4.

We propose a rowwise compression of $A_{B L}$ to construct a matrix $C$, referred to here as the communication matrix, which summarizes the communication requirement of row-parallel $y=A x$. First, for each $k=1, \ldots, K$, we compress the $k$ th row stripe into a single row with the sparsity pattern being equal to the union of the sparsities of all rows in that row stripe. Then, we discard the internal columns of $A_{B L}$ from the column set of $C$. Note that a nonzero entry $c_{k j}$ remains in $C$ if coupling column $j$ has at least one nonzero in the $k$ th row stripe. Therefore, rows of $C$ correspond to processors in such a way that the nonzeros in row $k$ identify the subset of $x_{C}$-vector entries needed by processor $P_{k}$. In other words, nonzeros in column $j$ of $C$ identify the set of processors that need $x_{C}[j]$. Since the columns of $C$ correspond to the coupling columns of $A_{B L}, C$ has $N_{C}=\left|x_{C}\right|$ columns, each of which has at least two nonzeros. Figure 3.1(a) illustrates communication matrix $C$ obtained from $A_{B L}$ shown in Figure 2.1(a). For example, the fourth row of matrix $C$ has nonzeros in columns $7,12,19,25$, and 26 corresponding to the nonzero coupling columns in the fourth row stripe of $A_{B L}$. These nonzeros summarize the need of processor $P_{4}$ for $x_{C}$-vector entries $x[7], x[12], x[19], x[25]$, and $x[26]$ in row-parallel $y=A x$.

Here, we exploit the row-net hypergraph model for sparse matrix representation $[7,8]$ to construct a communication hypergraph from matrix $C$. In this model, communication matrix $C$ is represented as a hypergraph $\mathcal{H}_{C}=(\mathcal{V}, \mathcal{N})$ on $N_{C}$ vertices and $K$ nets. Vertex and net sets $\mathcal{V}$ and $\mathcal{N}$ correspond to the columns and rows of matrix $C$, respectively. There exist one vertex $v_{j}$ for each column $j$ and one net $n_{k}$ for each row $k$. Consequently, vertex $v_{j}$ represents $x_{C}[j]$, and net $n_{k}$ represents processor $P_{k}$. Net $n_{k}$ contains vertices corresponding to the columns that have a nonzero in row $k$, i.e., $v_{j} \in n_{k}$ if and only if $c_{k j} \neq 0$. Nets $\left(v_{j}\right)$ contains the set of nets corresponding to the rows that have a nonzero in column $j$. In the proposed model, each vertex $v_{j}$ corresponds to the atomic task of expanding $x_{C}[j]$. Figure $3.1(\mathrm{c})$ shows 
the communication hypergraph obtained from the communication matrix $C$. In this figure, white and black circles represent, respectively, vertices and nets, and straight lines show the pins of nets.

3.1.2. Minimizing total latency and maximum volume. Here, we will show that minimizing the total latency and maintaining the balance on messagevolume loads of processors can be modeled as a hypergraph partitioning problem on the communication hypergraph. Consider a $K$-way partition $\Pi=\left\{\mathcal{V}_{1}, \ldots, \mathcal{V}_{K}\right\}$ of communication hypergraph $\mathcal{H}_{C}$. Without loss of generality, we assume that part $\mathcal{V}_{k}$ is assigned to processor $P_{k}$ for $k=1, \ldots, K$. The consistency of the proposed model for accurate representation of the total latency requirement depends on the condition that each net $n_{k}$ connects part $\mathcal{V}_{k}$ in $\Pi$, i.e., $\mathcal{V}_{k} \in \Lambda_{k}$. We first assume that this condition holds and discuss the appropriateness of the assumption later in section 3.4.

Since $\Pi$ is defined as a partition on the vertex set of $\mathcal{H}_{C}$, it induces a processor assignment for the atomic expand operations. Assigning vertex $v_{j}$ to part $\mathcal{V}_{\ell}$ is decoded as assigning the responsibility of expanding $x_{C}[j]$ to processor $P_{\ell}$. The destination set $\mathcal{E}_{j}$ in this expand operation is the set of processors corresponding to the nets that contain $v_{j}$ except $P_{\ell}$, i.e., $\mathcal{E}_{j}=N e t s\left(v_{j}\right)-\left\{P_{\ell}\right\}$. If $v_{j} \in n_{\ell}$, then $\left|\mathcal{E}_{j}\right|=d_{j}-1$; otherwise, $\left|\mathcal{E}_{j}\right|=d_{j}$. That is, the message-volume requirement of expanding $x_{C}[j]$ will be $d_{j}-1$ or $d_{j}$ words in the former and latter cases. Here, we prefer to associate a weight of $d_{j}-1$ with each vertex $v_{j}$ because the latter case is expected to be rare in partitionings. In this way, satisfying the partitioning constraint in (2.3) relates to maintaining the balance on message-volume loads of processors. Here, the messagevolume load of a processor refers to the volume of outgoing messages. We prefer to omit the incoming volume in considering the message-volume load of a processor with the assumption that each processor has enough local computation that overlaps with incoming messages in the network.

Consider a net $n_{k}$ with the connectivity set $\Lambda_{k}$ in partition $\Pi$. Let $\mathcal{V}_{\ell}$ be a part in $\Lambda_{k}$ other than $\mathcal{V}_{k}$. Also, let $v_{j}$ be a vertex of net $n_{k}$ in $\mathcal{V}_{\ell}$. Since $v_{j} \in \mathcal{V}_{\ell}$ and $v_{j} \in n_{k}$, processor $P_{\ell}$ will be sending $x_{C}[j]$ to processor $P_{k}$ due to the associated expand assignment. A similar send requirement is incurred by all other vertices of net $n_{k}$ in $\mathcal{V}_{\ell}$. That is, the vertices of net $n_{k}$ that lie in $\mathcal{V}_{\ell}$ show that $P_{\ell}$ must gather all $x_{C}$-vector entries corresponding to vertices in $n_{k} \cap \mathcal{V}_{\ell}$ into a single message to be sent to $P_{k}$. The size of this message will be $\left|n_{k} \cap \mathcal{V}_{\ell}\right|$ words. Hence, a net $n_{k}$ with the connectivity set $\Lambda_{k}$ shows that $P_{k}$ will be receiving a message from each processor in $\Lambda_{k}$ except itself. Hence, a net $n_{k}$ with the connectivity $\lambda_{k}$ shows $\lambda_{k}-1$ messages to be received by $P_{k}$ because $\mathcal{V}_{k} \in \Lambda_{k}$ (due to the consistency condition). The sum of the connectivity-1 values of all $K$ nets, i.e., $\sum_{n_{k}}\left(\lambda_{k}-1\right)$, will give the total number of messages received. As the total number of incoming messages is equal to the total number of outgoing messages, minimizing the objective function in (2.2) corresponds to minimizing the total message latency.

Figure 3.2(a) shows a partition of a generic communication hypergraph to clarify the above concepts. The main purpose of the figure is to show the number rather than the volume of messages, so multiple pins of a net in a part are contracted into a single pin. Arrows along the pins show the directions of the communication in the underlying expand operations. Figure 3.2(a) shows processor $P_{k}$ receiving messages from processors $P_{\ell}$ and $P_{m}$ because net $n_{k}$ connects parts $\mathcal{V}_{k}, \mathcal{V}_{\ell}$, and $\mathcal{V}_{m}$. The figure also shows $P_{k}$ sending messages to three different processors $P_{h}, P_{i}$, and $P_{j}$ due to nets $n_{h}, n_{i}$, and $n_{j}$ connecting part $\mathcal{V}_{k}$. Hence, the number of messages sent by $P_{k}$ is equal to $\left|\operatorname{Nets}\left(\mathcal{V}_{k}\right)\right|-1$. 


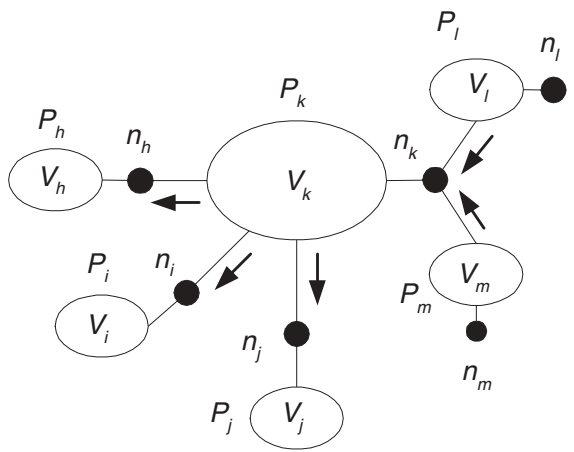

(a)

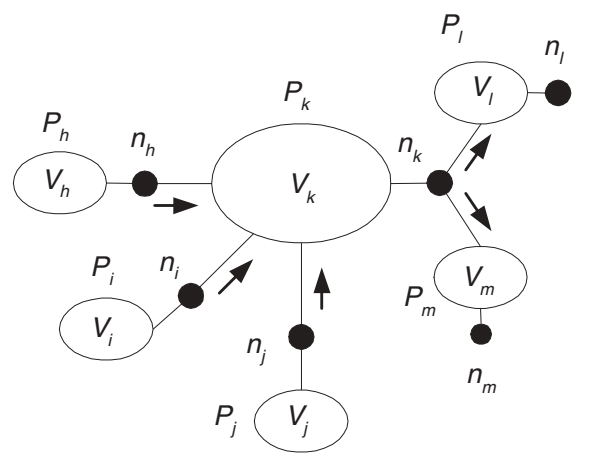

(b)

FIG. 3.2. Generic communication-hypergraph partitions for showing incoming and outgoing messages of processor $P_{k}$ in (a) row-parallel $y=A x$ and (b) column-parallel $w=A^{T} z$.

3.2. Column-parallel $\boldsymbol{w}=\boldsymbol{A}^{\boldsymbol{T}} \boldsymbol{z}$. Let $\left(A^{T}\right)_{B L}$ denote a block-structured form (see (2.1)) of $A^{T}$ for the given rowwise partition of $A$.

3.2.1. Communication-hypergraph model. A communication hypergraph for column-parallel $w=A^{T} z$ can be obtained from $\left(A^{T}\right)_{B L}$ as follows. We first determine the internal and coupling rows to form $w_{C}$, i.e., the $w$-vector entries that necessitate communication. We then apply a columnwise compression, similar to that in section 3.1.1, to obtain communication matrix $C^{T}$. Figure 3.1(b) illustrates communication matrix $C^{T}$ obtained from the block structure of $\left(A^{T}\right)_{B L}$ shown in Figure 2.1(b). Finally, we exploit the column-net hypergraph model for sparse matrix representation $[7,8]$ to construct a communication hypergraph from matrix $C^{T}$. The row-net and column-net hypergraph models are duals of each other. The column-net representation of a matrix is equivalent to the row-net representation of its transpose and vice versa. Therefore, the resulting communication hypergraph derived from $C^{T}$ will be topologically identical to that of the row-parallel $y=A x$ with dual communication-requirement association. For example, the communication hypergraph shown in Figure 3.1(c) represents communication matrix $C^{T}$ as well. In this hypergraph, net $n_{k}$ represents processor $P_{k}$ as before. However, vertices of net $n_{k}$ denote the set of $w_{C}$-vector entries for which processor $P_{k}$ generates partial results. Each vertex $v_{j}$ corresponds to the atomic task of folding on $w_{C}[j]$. Hence, Nets $\left(v_{j}\right)$ denotes the set of processors that generates a partial result for $w_{C}[j]$.

3.2.2. Minimizing total latency and maximum volume. Consider a $K$ way partition $\Pi=\left\{\mathcal{V}_{1}, \ldots, \mathcal{V}_{K}\right\}$ of communication hypergraph $\mathcal{H}_{C}$ with the same part-to-processor assignment and consistency condition as in section 3.1.2. Since the vertices of $\mathcal{H}_{C}$ correspond to fold operations, assigning a vertex $v_{j}$ to part $\mathcal{V}_{\ell}$ in $\Pi$ is decoded as assigning the responsibility of folding on $w_{C}[j]$ to processor $P_{\ell}$. Consider a net $n_{k}$ with the connectivity set $\Lambda_{k}$. Let $\mathcal{V}_{\ell}$ be a part in $\Lambda_{k}$ other than $\mathcal{V}_{k}$. Also, let $v_{j}$ be a vertex of net $n_{k}$ in $\mathcal{V}_{\ell}$. Since $v_{j} \in \mathcal{V}_{\ell}$ and $v_{j} \in n_{k}$, processor $P_{k}$ will be sending its partial result for $w_{C}[j]$ to $P_{\ell}$ because of the associated fold assignment to $P_{\ell}$. A similar send requirement is incurred to $P_{k}$ by all other vertices of net $n_{k}$ in $\mathcal{V}_{\ell}$. That is, the vertices of net $n_{k}$ that lie in $\mathcal{V}_{\ell}$ show that $P_{k}$ must gather all partial $w_{C}$ results corresponding to vertices in $n_{k} \cap \mathcal{V}_{\ell}$ into a single message to be sent to $P_{\ell}$. The size of this message will be $\left|n_{k} \cap \mathcal{V}_{\ell}\right|$ words. Hence, a net $n_{k}$ with connectivity set $\Lambda_{k}$ shows that $P_{k}$ will be sending a message to each processor in $\Lambda_{k}$ except itself. 
Hence, a net $n_{k}$ with the connectivity $\lambda_{k}$ shows $\lambda_{k}-1$ messages to be sent by $P_{k}$ because $\mathcal{V}_{k} \in \Lambda_{k}$ (due to the consistency condition). The sum of the connectivity-1 values of all $K$ nets, i.e., $\sum_{n_{k}}\left(\lambda_{k}-1\right)$, will give the total number of messages sent. Therefore, minimizing the objective function in (2.2) corresponds to minimizing the total message latency.

As vertices of $\mathcal{H}_{C}$ represent atomic fold operations, the weighted sum of vertices in a part will relate to the volume of incoming messages of the respective processor with vertex degree weighting. However, as mentioned earlier, we prefer to define the message-volume load of a processor as the volume of outgoing messages. Each vertex $v_{j}$ of net $n_{k}$ that lies in a part other than $\mathcal{V}_{k}$ incurs one word of message-volume load to processor $P_{k}$. In other words, each vertex of net $n_{k}$ that lies in part $\mathcal{V}_{k}$ relieves $P_{k}$ from sending a word. Thus, the message-volume load of $P_{k}$ can be computed in terms of the vertices in part $\mathcal{V}_{k}$ as $\left|n_{k}\right|-\left|n_{k} \cap \mathcal{V}_{k}\right|$. Here, we prefer to associate unit weights with vertices so that maintaining the partitioning constraint in (2.3) corresponds to an approximate message-volume load balancing. This approximation will prove to be a reasonable one if the net sizes are close to each other.

Figure 3.2(b) shows a partition of a generic communication hypergraph to illustrate the number of messages. Arrows along the pins of nets show the directions of messages for fold operations. Figure 3.2(b) shows processor $P_{k}$ sending messages to processors $P_{\ell}$ and $P_{m}$ because net $n_{k}$ connects parts $\mathcal{V}_{k}, \mathcal{V}_{\ell}$, and $\mathcal{V}_{m}$. Hence, the number of messages sent by $P_{k}$ is equal to $\lambda_{k}-1$.

3.3. Row-column-parallel $\boldsymbol{y}=\boldsymbol{A x}$ and $\boldsymbol{w}=\boldsymbol{A}^{\boldsymbol{T}} \boldsymbol{z}$. To minimize the total message count in $y=A x$ and $w=A^{T} z$, we use the same communication hypergraph $\mathcal{H}_{C}$ with different vertex weightings. As in sections 3.1.2 and 3.2.2, the cutsize of a partition of $\mathcal{H}_{C}$ quantifies the total number of messages sent both in $y=A x$ and $w=A^{T} z$. This property is in accordance with Facts 3 and 4 given in section 2.1.3. Therefore, minimizing the objective function in (2.2) corresponds to minimizing the total message count in row-column-parallel $y=A x$ and $w=A^{T} z$ multiplies.

Vertex weighting for maintaining the message-volume balance needs special attention. If there is a synchronization point between $w=A^{T} z$ and $y=A x$, the multiconstraint partitioning [25] should be adopted with two different weightings to impose a communication-volume balance in both multiply phases. If there is no synchronization point between the two multiplies (e.g., $y=A A^{T} z$ ), we recommend imposing a balance on aggregate message-volume loads of processors by associating an aggregate weight of $\left(d_{j}-1\right)+1=d_{j}$ with each vertex $v_{j}$.

3.4. Remarks on partitioning models. Consider a net $n_{k}$ which does not satisfy the consistency condition in a partition $\Pi$ of $\mathcal{H}_{C}$. Since $\mathcal{V}_{k} \notin \Lambda_{k}$, processor $P_{k}$ will be receiving a message from each processor in $\Lambda_{k}$ in row-parallel $y=A x$. Recall that $P_{k}$ needs the $x_{C}$-vector entries represented by the vertices in net $n_{k}$ independent of the connectivity between part $\mathcal{V}_{k}$ and net $n_{k}$. In a dual manner, $P_{k}$ will be sending a message to each processor in $\Lambda_{k}$ in column-parallel $w=A^{T} z$. Hence, net $n_{k}$ with the connectivity $\lambda_{k}$ will incur $\lambda_{k}$ incoming or outgoing messages instead of $\lambda_{k}-1$ messages determined by the cutsize of $\Pi$. That is, our model undercounts the actual number of messages by one for each net dissatisfying the consistency condition. In the worst case, this deviation may be as high as $K$ messages in total. This deficiency of the proposed model may be overcome by enforcing the consistency condition through exploiting the partitioning with fixed vertices feature, which exists in some of the hypergraph-partitioning tools $[1,9]$. We discuss such a method in section 4.1. 
Partitioning $x_{C}$-vector entries affects the message-volume requirement determined in the first phase. The message-volume requirement induced by the partitioning in the first phase is equal to $n n z(C)-N_{C}$ for row-parallel $y=A x$. Here, $n n z(C)$ and $N_{C}$ denote, respectively, the number of nonzeros and the number of columns in communication matrix $C$. Consider $x_{C}[j]$ corresponding to column $j$ of $C$. Assigning $x_{C}[j]$ to any one of the processors corresponding to the rows of $C$ that have a nonzero in column $j$ will not change the message-volume requirement. However, assigning it to some other processor will increase the message-volume requirement for expanding $x_{C}[j]$ by one word. In a partition $\Pi$ of communication hypergraph $\mathcal{H}_{C}$, this case corresponds to having a vertex $v_{j} \in \mathcal{V}_{k}$ while $v_{j} \notin n_{k}$. In other words, processor $P_{k}$ holds and expands $x_{C}[j]$ although it does not need it for local computations. A dual discussion holds for column-parallel $w=A^{T} z$, where such a vertex-to-part assignment corresponds to assigning the responsibility of folding on a particular $w_{C^{-}}$ vector entry to a processor which does not generate a partial result for that entry. In the worst case, the increase in the message volume may be as high as $N_{C}$ words in total for both types of multiplies. In hypergraph-theoretic view, the total message volume will be in between $\sum_{k}\left|n_{k}\right|-|\mathcal{V}|$ and $\sum_{k}\left|n_{k}\right|$, where $\sum_{k}\left|n_{k}\right|=n n z(C)$ and $|\mathcal{V}|=N_{C}$.

The proposed communication-hypergraph partitioning models exactly encode the total number of messages and the maximum message volume per processor metrics into the hypergraph partitioning objective and constraint, respectively, under the above conditions. The models do not directly encapsulate the metric of the maximum number of messages per processor; however, it is possible to address this metric within the partitioning framework. We give a method in section 4.3 to address this issue.

The allowed imbalance ratio $(\epsilon)$ is an important parameter in the proposed models. Choosing a large value for $\epsilon$ relaxes the partitioning constraint. Thus, large $\epsilon$ values enable the associated partitioning methods to achieve better partitioning objectives through enlarging the feasible search space. Hence, large $\epsilon$ values favor the total message-count metric. On the other hand, small $\epsilon$ values favor the maximum message-volume metric by imposing a tighter constraint on the part weights. Thus, $\epsilon$ should be chosen according to the target machine architecture and problem characteristics to trade the total latency for the maximum volume.

3.5. Illustration on the sample matrix. Figure 3.1(c) displays a four-way partition of the communication hypergraph, where closed dashed curves denote parts. Nets and their associated parts are kept close to each other. Note that the consistency condition is satisfied for the given partition. In the figure, net $n_{2}$ with the connectivity set $\Lambda_{2}=\left\{\mathcal{V}_{1}, \mathcal{V}_{2}, \mathcal{V}_{3}\right\}$ shows processor $P_{2}$ receiving messages from processors $P_{1}$ and $P_{3}$ in row-parallel $y=A x$. In a dual manner, net $n_{2}$ shows $P_{2}$ sending messages to $P_{1}$ and $P_{3}$ in column-parallel $w=A^{T} z$. Since the connectivities of nets $n_{1}, n_{2}, n_{3}$, and $n_{4}$ are, respectively, $2,3,3$, and 2 , the total message count is equal to $(2-1)+(3-1)+(3-$ $1)+(2-1)=6$ in both types of multiplies. Hence, the proposed approach reduces the number of messages from nine (see section 2.1.3) to six by yielding the given partition on $x_{C}$-vector $\left(w_{C}\right.$-vector) entries.

In the proposed two-phase approach, partitioning $x_{C}$-vector entries in the second phase can also be regarded as repermuting coupling columns of $A_{B L}$ obtained in the first phase. In a dual manner, partitioning $w_{C}$-vector entries can be regarded as repermuting coupling rows of $\left(A^{T}\right)_{B L}$. Figure 3.3 shows the repermuted $A_{B L}$ and $\left(A^{T}\right)_{B L}$ matrices induced by the sample communication-hypergraph partition shown in Figure 3.1(c). The total message count is 6 as enumerated by the total number 


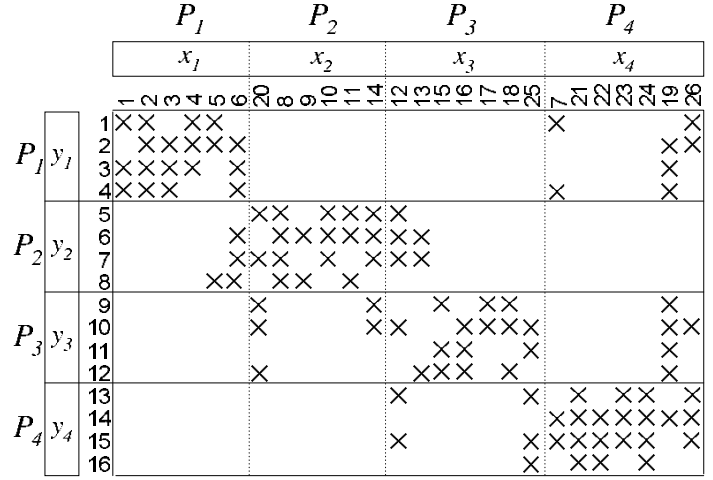

(a)

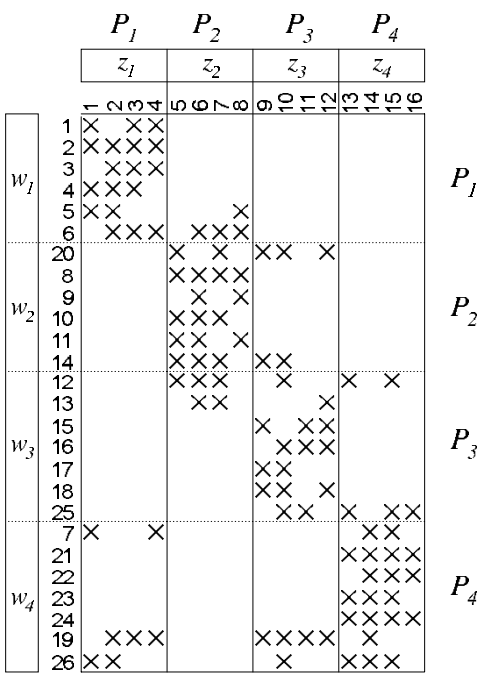

(b)

Fig. 3.3. Final $4 \times 4$ block structures: (a) $A_{B L}$ for row-parallel $y=A x$, and (b) $\left(A^{T}\right)_{B L}$ for column-parallel $w=A^{T} z$, induced by 4-way communication-hypergraph partition in Figure 3.1(c).

of nonzero off-diagonal blocks according to Fact 4, thus matching the cutsize of the partition given in Figure 3.1(c).

As seen in Figure 3.1(c), each vertex in each part is a pin of the net associated with that part. Therefore, for both types of multiplies, the sample partitioning does not increase the total message volume, and it remains at its lower bound which is $\sum_{k}\left|n_{k}\right|-|\mathcal{V}|=(5+6+7+5)-10=13$ words. This value can also be verified from the repermuted matrices given in Figure 3.3 by enumerating the total number of nonzero columns in the off-diagonal blocks according to Fact 4.

For row-parallel $y=A x$, the message-volume load estimates of processors are 2, 2,4 , and 5 words according to the vertex weighting proposed in section 3.1.2. These estimates are expected to be exact since each vertex in each part is a pin of the net associated with that part. This expectation can be verified from the repermuted $A_{B L}$ matrix given in Figure 3.3(a) by counting the number of nonzero columns in the off-diagonal blocks of the virtual column stripes according to Fact 1.

For column-parallel $w=A^{T} z$, the message-volume load estimates of processors are $2,2,3$, and 3 words according to the unit vertex weighting proposed in section 3.2.2. However, the actual message-volume loads of processors are 3, 4, 4, and 2 words. These values can be obtained from Figure 3.3(b) by counting the number of nonzero rows in the off-diagonal blocks of the virtual row stripes according to Fact 2. The above values yield an estimated imbalance ratio of $20 \%$ and an actual imbalance ratio of $23 \%$. The discrepancy between the actual and estimated imbalance ratios is because of the differences in net sizes.

4. Algorithms for communication-hypergraph partitioning. We present the following three methods for partitioning communication hypergraphs. In these methods, minimizing the cutsize while maintaining the partitioning constraint corresponds to minimizing the total number of messages while maintaining the balance on communication-volume loads of processors according to models proposed in sections 3.1.2 and 3.2.2. Method PaToH-fix is presented to show the feasibility of using 
a publicly available tool to partition communication hypergraphs. Method MSN involves some tailoring toward partitioning communication hypergraphs. Method $M S N \max$ tries to incorporate the minimization of the maximum message count per processor into the MSN method.

4.1. PaToH-fix: Recursive bipartitioning with fixed vertices. The multilevel paradigm has been successfully used in graph and hypergraph partitioning leading to successful tools $[9,17,22,24,26]$. The multilevel heuristics consist of three steps: coarsening, initial partitioning, and uncoarsening. In the first step, a multilevel clustering is applied starting from the original graph/hypergraph by adopting various matching/clustering heuristics until the number of vertices in the coarsened graph/hypergraph falls below a predetermined threshold. Clustering corresponds to coalescing highly interacting vertices of a level to supervertices of the next level. In the second step, a partition is obtained on the coarsest graph/hypergraph using various heuristics. In the third step, the partition found in the second step is successively projected back toward the original graph/hypergraph by refining the projected partitions on the intermediate level uncoarser graphs/hypergraphs using various heuristics. A common refinement heuristic is FM, which is an iterative improvement method proposed for graph/hypergraph bipartitioning by Fiduccia and Mattheyses [13] as a faster implementation of the KL algorithm proposed by Kernighan and Lin [27].

In this work, we use the multilevel hypergraph-partitioning tool $\mathrm{PaToH}$ [9] for partitioning communication hypergraphs. Recall that the communication-hypergraph partitioning differs from the conventional hypergraph partitioning because of the netto-part association needed to satisfy the consistency condition mentioned in sections 3.1.2 and 3.2.2. We exploit the partitioning with fixed vertices feature supported by $\mathrm{PaToH}$ to achieve this net-to-part association as follows. The communication hypergraph is augmented with $K$ zero-weighted artificial vertices of degree one. Each artificial vertex $v_{k}^{*}$ is added to a unique net $n_{k}$ as a new pin and marked as fixed to part $\mathcal{V}_{k}$. This augmented hypergraph is fed to $\mathrm{PaToH}$ for $K$-way partitioning. $\mathrm{PaToH}$ generates $K$-way partitions with these $K$ labeled vertices lying in their fixed parts thus establishing the required net-to-part association. A $K$-way partition $\Pi=$ $\left\{\mathcal{V}_{1}, \ldots, \mathcal{V}_{K}\right\}$ generated by $\mathrm{PaToH}$ is decoded as follows. The atomic communication tasks associated with the actual vertices assigned to part $\mathcal{V}_{k}$ are assigned to processor $P_{k}$, whereas $v_{k}^{*}$ does not incur any communication task.

4.2. MSN: Direct $K$-way partitioning. Most of the partitioning tools, including $\mathrm{PaToH}$, achieve $K$-way partitioning through recursive bisection. In this scheme, first a two-way partition is obtained, and then this two-way partition is further bipartitioned recursively. The connectivity-1 cutsize metric (see (2.2)) is easily handled through net splitting [8] during recursive bisection steps. Although the recursive-bisection paradigm is successful in $K$-way partitioning in general, its performance degrades for hypergraphs with large net sizes. Since communication hypergraphs have nets with large sizes, this degradation is also expected to be notable with $\mathrm{PaToH}$-fix. In order to alleviate this problem, we have developed a multilevel direct $K$-way hypergraph partitioner $(M S N)$ by integrating Sanchis's direct $K$-way refinement (SN) algorithm [35] to the uncoarsening step of the multilevel framework.

The coarsening step of $M S N$ is essentially the same as that of PaToH. In the initial partitioning step, a $K$-way partition on the coarsest hypergraph is obtained by using a simple constructive approach which mainly aims to satisfy the balance constraint. In $M S N$, the net-to-part association is handled implicitly rather than by introducing artificial vertices. This association is established in the initial partitioning step through 
associating each part with a distinct net which connects that part, and it is maintained later in the uncoarsening step. In the uncoarsening step, the SN algorithm, which is a generalization of the two-way FM paradigm to $K$-way refinement $[11,36]$, is used. SN, starting from a $K$-way initial partition, performs a number of passes until it finds a locally optimum partition, where each pass consists of a sequence of vertex moves. The fundamental idea is the notion of gain, which is the decrease in the cutsize of a partition due to a vertex moving from a part to another. The local search strategy adopted in the SN approach repeatedly moves a vertex with the maximum gain even if that gain is negative and records the best partition encountered during a pass. Allowing tentative moves with negative gains brings restricted "hill-climbing ability" to the approach.

In the SN algorithm, there are $K-1$ possible moves for each vertex. The algorithm stores the gains of the moves from a source part in $K-1$ associated priority queuesone for each possible destination part. Hence, the algorithm uses $K(K-1)$ priority queues with a space complexity of $O\left(N_{C} K\right)$, which may become a memory problem for large $K$. The moves with the maximum gain are selected from each of these $K(K-1)$ priority queues, and the one that maintains the balance criteria is performed. After the move, only the move gains of the vertices that share a net with the moved vertex may need to be updated. This may lead to updates on at most $4 K-6$ priority queues. Within a pass, a vertex is allowed to move at most once.

4.3. MSNmax: Considering maximum message latency. The proposed models do not encapsulate the minimization of the maximum message latency per processor. By similar reasoning in defining the message-volume load of a processor as the volume of outgoing messages, we prefer to define the message-latency load of a processor in terms of the number of outgoing messages. Here, we propose a practical way of incorporating the minimization of the maximum message-count metric into the MSN method. The resulting method is referred to here as MSNmax. MSNmax differs from $M S N$ only in the SN refinement scheme used in the uncoarsening phase. $M S N \max$ still relies on the same gain notion and maintains updated move gains in $K(K-1)$ priority queues. The difference lies in the move selection policy, which favors the moves that reduce the message counts of overloaded processors. Here, a processor is said to be overloaded if its message count is above the average by a prescribed percentage (e.g., $25 \%$ is used in this work). For this purpose, message counts of processors are maintained during the course of the SN refinement algorithm.

For row-parallel $y=A x$, the message count of a processor can be reduced by moving vertices out of the associated part. Recall that moving a vertex from a part corresponds to relieving the associated processor of the respective atomic expand task. For this reason, only the priority queues of the overloaded parts are considered for selecting the move with the maximum gain. For column-parallel $w=A^{T} z$, the message count of a processor $P_{k}$ can be reduced by reducing the connectivity of the associated net $n_{k}$ through moves from the parts in $\Lambda_{k}-\left\{P_{k}\right\}$. Therefore, only the priority queues of the parts that are in the connectivity sets of the nets associated with the overloaded parts are considered. For both types of parallel multiplies, moves selected from the restricted set of priority queues are likely to decrease the message counts of overloaded processors besides decreasing the total message count.

5. Experimental results. We have tested the performance of the proposed models and associated partitioning methods on a wide range of large unsymmetric square and rectangular sparse matrices. Properties of these matrices are listed in Table 5.1. The first four matrices, which are obtained from University of Florida 
TABLE 5.1

Properties of unsymmetric square and rectangular test matrices.

\begin{tabular}{|c|c|c|c|c|c|c|c|c|c|}
\hline \multicolumn{4}{|c|}{$M \times N$ matrix $A$} & \multicolumn{6}{|c|}{$K \times N_{C}$ communication matrix $C$} \\
\hline & & & & & 24 & & 64 & $K=$ & 128 \\
\hline Name & $M$ & $N$ & $N N Z$ & $N_{C}$ & $N N Z$ & $N_{C}$ & $N N Z$ & $N_{C}$ & $N N Z$ \\
\hline lhr14 & 14270 & 14270 & 321988 & 11174 & 25188 & 12966 & 31799 & 13508 & 36039 \\
\hline lhr17 & 17576 & 17576 & 399500 & 13144 & 29416 & 16070 & 38571 & 16764 & 46182 \\
\hline onetone1 & 36057 & 36057 & 368055 & 8137 & 20431 & 11458 & 30976 & 13911 & 39936 \\
\hline onetone 2 & 36057 & 36057 & 254595 & 3720 & 9155 & 6463 & 16259 & 11407 & 27264 \\
\hline pig-large & 28254 & 17264 & 75018 & 1265 & 3347 & 1522 & 4803 & 1735 & 6193 \\
\hline pig-very & 174193 & 105882 & 463303 & 4986 & 12015 & 6466 & 16185 & 7632 & 20121 \\
\hline CO9 & 10789 & 14851 & 101578 & 4458 & 9226 & 7431 & 21816 & 7887 & 25070 \\
\hline fxm4-6 & 22400 & 30732 & 248989 & 769 & 1650 & 2010 & 4208 & 4223 & 8924 \\
\hline kent & 31300 & 16620 & 184710 & 5200 & 10691 & 11540 & 28832 & 14852 & 49976 \\
\hline $\bmod 2$ & 34774 & 31728 & 165129 & 4760 & 9870 & 8634 & 18876 & 10972 & 24095 \\
\hline pltexpA4 & 26894 & 70364 & 143059 & 1961 & 4218 & 3259 & 7858 & 5035 & 13397 \\
\hline world & 34506 & 32734 & 164470 & 5116 & 10405 & 9569 & 20570 & 13610 & 30881 \\
\hline
\end{tabular}

Sparse Matrix Collection, ${ }^{1}$ are from the unsymmetric linear system application. The pig-large and pig-very matrices [18] are from the least squares problem. The remaining six matrices, which are obtained from Hungarian Academy of Sciences OR $\mathrm{Lab},{ }^{2}$ are from miscellaneous and stochastic linear programming problems. In this table, the $N N Z$ column lists the number of nonzeros of the matrices.

We have tested $K=24-, 64-$, and 128-way rowwise partitionings of each test matrix. For each $K$ value, $K$-way partitioning of a test matrix forms a partitioning instance. Recall that the objective in the first phase of our two-phase approach is minimizing the total message volume while maintaining the computational-load balance. This objective is achieved by exploiting the recently proposed computationalhypergraph model [8]. The hypergraph-partitioning tool PaToH [9] was used with default parameters to obtain $K$-way rowwise partitions. The computational-load imbalance values of all partitions were measured to be below $6 \%$.

For the second phase, communication matrix $C$ was constructed for every partitioning instance as described in sections 3.1.1 and 3.2.1. Table 5.1 displays properties of these communication matrices. Then, the communication hypergraph was constructed from each communication matrix as described in sections 3.1.1 and 3.2.1. Note that communication-matrix properties listed in Table 5.1 also show communication-hypergraph properties. That is, for each $K$ value, the table effectively shows a communication hypergraph on $K$ nets, $N_{C}$ vertices, and $N N Z$ pins.

The communication hypergraphs are partitioned using the proposed methods discussed in section 4 . In order to verify the validity of the communication hypergraph model, we compare the performance of these methods with a method called Naive. This method mimics the current state of the art by minimizing the communication overhead due to the message volume without spending any explicit effort toward minimizing the total message count. The Naive method tries to obtain a balance on message-volume loads of processors while attaining the total message-volume requirement determined by the partitioning in the first phase. The method adopts a constructive approach, which is similar to the best-fit-decreasing heuristic used in solving the NP-hard $K$-feasible bin packing problem [23]. Vertices of the communication hypergraph are assigned to parts in the decreasing order of vertex weights. Each

\footnotetext{
${ }^{1}$ http://www.cise.ufl.edu/ davis/sparse/

${ }^{2} \mathrm{ftp}: / /$ ftp.sztaki.hu/pub/oplab
} 
TABLE 5.2

Performance of the methods with varying imbalance ratios in 64-way partitionings.

\begin{tabular}{ll|rrrr|rrrr}
\hline Matrix & Partition & \multicolumn{4}{|c|}{ Total msg } & \multicolumn{4}{c}{ Max vol } \\
\cline { 3 - 10 } & method & $\epsilon=0.1$ & $\epsilon=0.3$ & $\epsilon=0.5$ & $\epsilon=1.0$ & $\epsilon=0.1$ & $\epsilon=0.3$ & $\epsilon=0.5$ & $\epsilon=1.0$ \\
\hline lhr17 & Naive & 1412 & - & - & - & 373 & - & - & - \\
& PaToHfix & 817 & 726 & 724 & 700 & 643 & 755 & 858 & 1042 \\
& MSN & 745 & 662 & 625 & 592 & 678 & 793 & 895 & 1177 \\
& MSNmax & 731 & 684 & 649 & 638 & 676 & 799 & 920 & 1119 \\
\hline pig-very & Naive & 2241 & - & - & - & 161 & - & - & - \\
& PaToHfix & 1333 & 1176 & 1151 & 1097 & 272 & 316 & 361 & 448 \\
& MSN & 1407 & 1199 & 1137 & 1019 & 284 & 343 & 398 & 526 \\
& MSNmax & 1293 & 1142 & 1040 & 967 & 298 & 354 & 411 & 530 \\
\hline fxm4-6 & Naive & - & - & - & 312 & - & - & - & 67 \\
& PaToHfix & 212 & 193 & 193 & 188 & 70 & 75 & 81 & 105 \\
& MSN & 244 & 205 & 199 & 172 & 72 & 83 & 96 & 114 \\
& MSNmax & 247 & 213 & 208 & 165 & 70 & 85 & 94 & 103 \\
\hline
\end{tabular}

vertex $v_{j}$ is allowed to be assigned only to the parts in $N e t s\left(v_{j}\right)$ to avoid increases in the message volume. Here, the best-fit criterion corresponds to assigning $v_{j}$ to a part in $N e t s\left(v_{j}\right)$ with the minimum weight thus trying to obtain a balance on the message-volume loads.

The partitioning methods, PaToH-fix, MSN, and MSNmax, incorporate randomized algorithms. Therefore, they were run 20 times starting from different random seeds for $K$-way partitioning of every communication hypergraph. Randomization in the Naive method were realized by random permutation of the vertices before sorting. Averages of the resulting communication patterns of these runs are displayed in the following tables. In these tables, the Total msg and Total vol columns list, respectively, the total number and total volume of messages sent. The Max msg and Max vol columns list, respectively, the maximum number and maximum volume of messages sent by a single processor.

The following parameters and options are used in the proposed partitioning methods. PaToH-fix were run with the coarsening option of absorption clustering using pins (ABS_HPC), and the refinement option of Fiduccia-Mattheyses (FM). The scaled heavy-connectivity matching (SHCM) of $\mathrm{PaToH}$ was used in the coarsening step of the multilevel partitioning methods $M S N$ and MSNmax. ABS_HPC is the default coarsening option in PaToH-fix. It is a quite powerful coarsening method that absorbs nets into supervertices, which helps FM-based recursive-bisection heuristics. However, we do not want nets being absorbed in $M S N$ and $M S N \max$ to be able to establish netto-part association in the initial partitioning phase. Therefore, SHCM, which does not aim to absorb nets, was selected.

Table 5.2 shows the performance of the proposed methods with varying $\epsilon$ in 64-way partitioning of three matrices, each of which is the largest (in terms of the number of nonzeros) in its application domain. The performance variation is displayed in terms of the total message-count and maximum message-volume metrics because these two metrics are exactly encoded in the proposed models. Recall that Naive is a constructive method and its performance does not depend on $\epsilon$. Therefore, the performance values for Naive are listed under the columns corresponding to the attained imbalance ratios. As seen in Table 5.2, by relaxing $\epsilon$, each method can find partitions with smaller total message counts and larger maximum message-volume values. It is also observed that imbalance values of the partitions obtained by all of the proposed methods are usually very close to the given $\epsilon$. These outcomes are in accordance 
with the discussion in section 3.4. As seen in the table, all of the proposed methods perform significantly better than the Naive method even with the tightest constraint of $\epsilon=0.1$. However, the detailed performance results are displayed for $\epsilon=1.0$ (i.e., $W_{\max } \leq 2 W_{\text {avg }}$ in (2.3)) in the following tables. We chose such a relaxed partitioning constraint in order to discriminate among the proposed methods. It should be noted here that imbalance ratios for the message-volume loads of processors might be greater than the chosen $\epsilon$ value because of the approximation in the proposed vertex weighting scheme. For example, with $\epsilon=1.0$, the methods PaToH-fix, MSN, and MSNmax produce partitions with actual imbalance ratios of $0.94,1.26$, and 1.35 for matrix $\mathrm{lhr} 17$, respectively.

Table 5.3 displays the communication patterns for $K=64$ - and 128-way partitions in row-parallel $y=A x$. The bottom of the table shows the average performance of the proposed methods compared with the Naive method. These values are obtained by first normalizing the performance results of the proposed methods with respect to those of the Naive method for every partitioning instance and then averaging these normalized values over the individual methods.

In terms of the total message-volume metric, Naive achieves the lowest values as seen in Table 5.3. This is expected since Naive attains the total message volume determined by the partitioning in the first phase. The increase in the total message-volume values for the proposed methods remain below $66 \%$ for all partitioning instances. As seen in the bottom of the table, these increases are below $41 \%$ on the average. Note that the total message-volume values for Naive are equal to the differences of the $N N Z$ and $N_{C}$ values of the respective communication matrix (see Table 5.1). Also note that the $N N Z$ values of the communication matrices listed in Table 5.1 show the upper bounds on the total message-volume values for the proposed partitioning methods.

In terms of the maximum message-volume metric, the proposed partitioning methods yield worse results than the Naive method by a factor between 2.0 and 2.4 on the average as seen in the bottom of Table 5.3. This performance difference stems from three factors. First, Naive is likely to achieve small maximum message-volume values since it achieves the lowest total message-volume values. Second, the best-fitdecreasing heuristic adopted in Naive is an explicit effort toward achieving a balance on the message volume. Third, the relaxed partitioning constraint $(\epsilon=1.0)$ used in the proposed partitioning methods leads to higher imbalance ratios among the message-volume loads of processors.

In terms of the total message-count metric, all of the proposed methods yield significantly better results than the Naive method in all partitioning instances. They reduce the total message count by a factor between 1.3 and 3.0 in 64-way, and between 1.2 and 2.9 in 128-way partitionings. As seen in the bottom of Table 5.3, the reduction factor is approximately 2 on the average. Comparing the performance of the proposed methods, both MSN and MSNmax perform better than PaToH-fix in all partitioning instances, except 64-way partitioning of plexpA_4 and 128-way partitioning of onetone2, leading to a considerable performance difference on the average. This experimental finding confirms the superiority of the direct $K$-way partitioning approach over the recursive-bisection approach. There is no clear winner between $M S N$ and MSNmax. MSN performs better than MSNmax in 14 out of 24 partitioning instances, leading to a slight performance difference on the average.

In terms of the maximum message-count metric, all of the proposed methods again yield considerably better results than the Naive method in all instances, except 64- and 128-way partitionings of pig matrices. However, the performance difference 
TABLE 5.3

Communication patterns for $K$-way row-parallel $y=A x$.

\begin{tabular}{|c|c|c|c|c|c|c|c|c|c|}
\hline \multirow{3}{*}{ Matrix } & \multirow{3}{*}{$\begin{array}{l}\text { Part. } \\
\text { method }\end{array}$} & \multicolumn{4}{|c|}{$K=64$} & \multicolumn{4}{|c|}{$K=128$} \\
\hline & & \multicolumn{2}{|c|}{ Total } & \multicolumn{2}{|c|}{$\operatorname{Max}$} & \multicolumn{2}{|c|}{ Total } & \multicolumn{2}{|c|}{$\operatorname{Max}$} \\
\hline & & msg & vol & msg & vol & $\mathrm{msg}$ & vol & msg & vol \\
\hline \multirow[t]{4}{*}{ lhr14 } & Naive & 1318 & 18833 & 43.9 & 308 & 2900 & 22531 & 47.6 & 204 \\
\hline & PaToH-fix & 676 & 28313 & 34.0 & 813 & 1417 & 32661 & 47.8 & 627 \\
\hline & MSN & 561 & 26842 & 24.4 & 975 & 1247 & 30796 & 31.6 & 577 \\
\hline & MSNmax & 640 & 24475 & 19.2 & 897 & 1348 & 28758 & 22.7 & 535 \\
\hline \multirow[t]{4}{*}{ lhr17 } & Naive & 1412 & 22501 & 45.6 & 373 & 3675 & 29418 & 58.9 & 265 \\
\hline & PaToH-fix & 700 & 34515 & 36.5 & 1042 & 1867 & 42623 & 54.3 & 750 \\
\hline & MSN & 592 & 32530 & 26.3 & 1177 & 1453 & 40009 & 34.0 & 736 \\
\hline & MSNmax & 638 & 31149 & 22.0 & 1119 & 1599 & 38557 & 26.8 & 689 \\
\hline \multirow[t]{4}{*}{ onetone1 } & Naive & 1651 & 19518 & 39.9 & 332 & 4112 & 26025 & 47.4 & 231 \\
\hline & РaToH-fix & 663 & 26789 & 27.2 & 714 & 1639 & 35741 & 39.1 & 580 \\
\hline & MSN & 545 & 27109 & 24.1 & 1008 & 1384 & 35129 & 31.1 & 688 \\
\hline & MSNmax & 610 & 24012 & 20.9 & 950 & 1507 & 31345 & 26.4 & 642 \\
\hline \multirow[t]{4}{*}{ onetone 2} & Naive & 995 & 9796 & 30.4 & 186 & 2049 & 15857 & 28.6 & 139 \\
\hline & PaToH-fix & 429 & 12940 & 17.8 & 381 & 804 & 20983 & 25.1 & 423 \\
\hline & MSN & 406 & 13236 & 17.1 & 510 & 787 & 20649 & 22.1 & 422 \\
\hline & MSNmax & 420 & 12389 & 15.1 & 485 & 807 & 18850 & 20.4 & 381 \\
\hline \multirow{4}{*}{ pig-large } & Naive & 1220 & 3281 & 39.4 & 60 & 2723 & 4458 & 39.6 & 47 \\
\hline & PaToH-fix & 759 & 4363 & 40.5 & 144 & 1764 & 5805 & 52.5 & 142 \\
\hline & MSN & 619 & 4108 & 34.5 & 153 & 1551 & 5752 & 43.0 & 115 \\
\hline & MSNmax & 682 & 3812 & 35.6 & 138 & 1678 & 5185 & 35.0 & 100 \\
\hline \multirow[t]{4}{*}{ pig-very } & Naive & 2241 & 9719 & 56.5 & 161 & 4574 & 12489 & 78.7 & 117 \\
\hline & PaToH-fix & 1097 & 14725 & 59.8 & 448 & 2533 & 18567 & 97.8 & 398 \\
\hline & MSN & 1019 & 14349 & 54.5 & 526 & 2389 & 17317 & 77.3 & 320 \\
\hline & MSNmax & 967 & 14008 & 55.4 & 530 & 2501 & 15729 & 80.5 & 317 \\
\hline \multirow[t]{4}{*}{ CO9 } & Naive & 1283 & 14385 & 41.0 & 369 & 1645 & 17183 & 48.9 & 289 \\
\hline & PaToH-fix & 622 & 19221 & 34.6 & 567 & 1191 & 23575 & 35.8 & 434 \\
\hline & MSN & 521 & 18352 & 27.1 & 687 & 904 & 20727 & 28.9 & 412 \\
\hline & MSNmax & 513 & 17736 & 23.1 & 684 & 800 & 21281 & 25.6 & 492 \\
\hline \multirow[t]{4}{*}{ fxm4-6 } & Naive & 312 & 2198 & 13.6 & 67 & 562 & 4701 & 15.9 & 64 \\
\hline & PaToH-fix & 188 & 2856 & 11.8 & 105 & 361 & 5746 & 13.8 & 129 \\
\hline & MSN & 172 & 2746 & 10.1 & 114 & 338 & 5647 & 12.2 & 129 \\
\hline & MSNmax & 165 & 2543 & 8.9 & 103 & 322 & 5386 & 11.7 & 124 \\
\hline kent & Naive & 342 & 17292 & 14.1 & 547 & 1020 & 35124 & 21.9 & 602 \\
\hline & PaToH-fix & 235 & 21200 & 9.2 & 621 & 740 & 42328 & 15.8 & 631 \\
\hline & MSN & 190 & 21539 & 8.9 & 905 & 596 & 39774 & 19.6 & 866 \\
\hline & MSNmax & 201 & 19666 & 7.0 & 773 & 614 & 40012 & 13.0 & 830 \\
\hline $\bmod 2$ & Naive & 376 & 10242 & 22.4 & 366 & 811 & 13123 & 33.8 & 240 \\
\hline & PaToH-fix & 294 & 16683 & 19.8 & 606 & 658 & 21409 & 22.6 & 431 \\
\hline & MSN & 254 & 13353 & 15.2 & 604 & 575 & 17329 & 18.7 & 391 \\
\hline & MSNmax & 231 & 14400 & 12.5 & 639 & 548 & 19009 & 14.4 & 408 \\
\hline pltexpA4 & Naive & 507 & 4599 & 21.9 & 116 & 1013 & 8362 & 25.6 & 99 \\
\hline & РaToH-fix & 257 & 5553 & 17.7 & 243 & 579 & 10163 & 22.8 & 208 \\
\hline & MSN & 245 & 5828 & 15.3 & 241 & 556 & 9705 & 21.7 & 213 \\
\hline & MSNmax & 264 & 5321 & 13.2 & 214 & 546 & 9582 & 19.4 & 206 \\
\hline world & Naive & 534 & 11001 & 27.4 & 387 & 1785 & 17271 & 44.1 & 222 \\
\hline & РaToH-fix & 362 & 18355 & 21.2 & 603 & 1036 & 26514 & 35.6 & 488 \\
\hline & MSN & 315 & 14765 & 16.8 & 595 & 902 & 23927 & 24.8 & 476 \\
\hline & MSNmax & 287 & 16243 & 14.8 & 680 & 886 & 23762 & 20.6 & 476 \\
\hline & & & & ormal & ed ave & ges $\mathrm{O}$ & Naive & & \\
\hline & Naive & 1.00 & 1.00 & 1.00 & 1.00 & 1.00 & 1.00 & 1.00 & 1.00 \\
\hline & РaToH-fix & 0.56 & 1.41 & 0.81 & 2.03 & 0.59 & 1.38 & 0.91 & 2.38 \\
\hline & MSN & 0.48 & 1.34 & 0.68 & 2.37 & 0.51 & 1.29 & 0.75 & 2.30 \\
\hline & MSNmax & 0.49 & 1.28 & 0.60 & 2.26 & 0.52 & 1.24 & 0.63 & 2.21 \\
\hline
\end{tabular}


between the proposed methods and the Naive method is not as large as that in the total message-count metric. Comparing the performance of the proposed methods, both $M S N$ and MSNmax perform better than $\mathrm{PaToH}$-fix in all partitioning instances, except 128-way partitioning of kent, leading to a considerable performance difference on the average. MSNmax is the clear winner in the maximum message-count metric as expected. As seen in the bottom of the table, MSNmax yields, respectively, $40 \%$ and $37 \%$ fewer maximum message counts than Naive, for 64 and 128-way partitionings, on the average.

We have also experimented with the performance of the proposed methods for 64-way and 128-way partitionings for column-parallel $w=A^{T} z$ and row-columnparallel $y=A A^{T} z$ on the test matrices. Since very similar relative performance results were obtained in these experiments, we omit presentation and discussion of these experimental results due to the lack of space.

It is important to see whether the theoretical improvements obtained by our methods in the given performance metrics hold in practice. For this purpose, we have implemented row-parallel $y=A x$ and row-column-parallel $y=A A^{T} z$ multiplies using the LAM/MPI 6.5.6 [5] message passing library. The parallel multiply programs were run on a Beowulf class [38] PC cluster with 24 nodes. Each node has a 400Mhz Pentium-II processor and 128MB memory. The interconnection network is comprised of a 3COM SuperStack II 3900 managed switch connected to Intel Ethernet Pro 100 Fast Ethernet network interface cards at each node. The system runs the Linux kernel 2.4.14 and the Debian GNU/Linux 3.0 distribution.

Within the current experimental framework, MSNmax seems to be the best choice for communication-hypergraph partitioning. For this reason, in Table 5.4, only the parallel running times of the multiply programs for MSNmax partitionings are given in comparison with those for Naive partitionings. Communication patterns for the resulting partitions are also listed in the table in order to show how improvements in performance metrics relate to improvements in parallel running times.

As seen in Table 5.4, the partitions obtained by MSNmax lead to considerable improvements in parallel running times compared with those of Naive for all matrices. The improvements in parallel running times are in between $4 \%$ and $40 \%$ in $y=A x$, and between $5 \%$ and $31 \%$ in $y=A A^{T} z$. In row-parallel $y=A x$, the lowest percent improvement of $4 \%$ occurs for matrix kent despite the modest improvement of $28 \%$ achieved by MSNmax over Naive in total message count. The reason seems to be the equal maximum message counts obtained by these partitioning methods. The highest percent improvement of $40 \%$ occurs for matrix fxm4-6 for which MSNmax achieves significant improvements of $49 \%$ and $36 \%$ in the total and maximum message counts, respectively. However, the higher percent improvements obtained by MSNmax for matrix 1 hr14 in message-count metrics do not lead to higher percent improvements in parallel running time. This might be attributed to MSNmax achieving lower percent improvements for $\mathrm{lhr} 14$ in message-volume metrics compared with those for fxm4-6. These experimental findings confirm the difficulty of the target problem.

Table 5.5 displays partitioning times for the three largest matrices selected from different application domains. The Phase 1 Time and Phase 2 Time columns list, respectively, the computational-hypergraph and communication-hypergraph partitioning times. Sequential matrix-vector multiply times are also displayed to show the relative preprocessing overhead introduced by the partitioning methods. All communication-hypergraph partitionings take significantly less time than computational-hypergraph partitionings except partitioning communication hypergraph of 
TABLE 5.4

Communication patterns and parallel running times in msecs for 24-way row-parallel $y=A x$ and row-column-parallel $y=A A^{T} z$.

\begin{tabular}{|c|c|c|c|c|c|c|c|c|c|c|c|}
\hline \multirow{3}{*}{ Matrix } & \multirow{3}{*}{$\begin{array}{l}\text { Part. } \\
\text { method }\end{array}$} & \multicolumn{5}{|c|}{$y=A x$} & \multicolumn{5}{|c|}{$y=A A^{T} z$} \\
\hline & & \multicolumn{2}{|c|}{ Total } & \multicolumn{2}{|c|}{ Max } & \multirow{2}{*}{$\begin{array}{l}\text { Parl } \\
\text { time }\end{array}$} & \multicolumn{2}{|c|}{ Total } & \multicolumn{2}{|c|}{ Max } & \multirow{2}{*}{$\begin{array}{l}\text { Parl } \\
\text { time }\end{array}$} \\
\hline & & msg & vol & msg & vol & & msg & vol & msg & vol & \\
\hline \multirow[t]{2}{*}{ lhr14 } & Naive & 414 & 14014 & 23 & 603 & 2.57 & 838 & 28028 & 46 & 1177 & 5.07 \\
\hline & MSNmax & 176 & 19580 & 12 & 1601 & 1.90 & 342 & 42456 & 27 & 1960 & 3.95 \\
\hline \multirow[t]{2}{*}{ lhr17 } & Naive & 393 & 16272 & 22 & 691 & 2.79 & 792 & 32544 & 45 & 1159 & 5.71 \\
\hline & MSNmax & 168 & 24510 & 17 & 2229 & 2.20 & 334 & 48554 & 23 & 2112 & 4.38 \\
\hline \multirow[t]{2}{*}{ onetone1 } & Naive & 362 & 12294 & 19 & 546 & 2.52 & 728 & 24588 & 41 & 788 & 5.49 \\
\hline & MSNmax & 152 & 15153 & 16 & 1403 & 1.85 & 262 & 34304 & 24 & 1586 & 4.37 \\
\hline \multirow[t]{2}{*}{ onetone 2} & Naive & 205 & 5435 & 12 & 297 & 1.60 & 412 & 10870 & 24 & 419 & 3.24 \\
\hline & MSNmax & 102 & 6294 & 9 & 690 & 1.31 & 186 & 15234 & 16 & 715 & 2.44 \\
\hline \multirow[t]{2}{*}{ pig-large } & Naive & 325 & 2082 & 23 & 108 & 2.06 & 650 & 4164 & 42 & 162 & 3.41 \\
\hline & MSNmax & 151 & 2872 & 20 & 276 & 1.28 & 312 & 5554 & 26 & 271 & 2.35 \\
\hline \multirow[t]{2}{*}{ pig-very } & Naive & 497 & 7029 & 23 & 354 & 3.51 & 994 & 14058 & 46 & 456 & 7.33 \\
\hline & MSNmax & 228 & 10214 & 23 & 937 & 2.74 & 428 & 20538 & 29 & 963 & 5.95 \\
\hline \multirow[t]{2}{*}{ CO9 } & Naive & 122 & 4768 & 11 & 437 & 1.74 & 244 & 9536 & 22 & 1184 & 3.34 \\
\hline & MSNmax & 68 & 6834 & 9 & 750 & 1.35 & 152 & 13700 & 16 & 1430 & 2.99 \\
\hline \multirow[t]{2}{*}{ fxm4-6 } & Naive & 113 & 881 & 11 & 44 & 1.57 & 226 & 1762 & 27 & 108 & 3.18 \\
\hline & MSNmax & 58 & 1005 & 7 & 96 & 0.95 & 120 & 2038 & 15 & 124 & 2.31 \\
\hline \multirow[t]{2}{*}{ kent } & Naive & 57 & 5491 & 5 & 488 & 1.12 & 114 & 10982 & 9 & 972 & 2.27 \\
\hline & MSNmax & 41 & 5783 & 5 & 541 & 1.08 & 86 & 12596 & 7 & 1025 & 2.12 \\
\hline \multirow[t]{2}{*}{$\bmod 2$} & Naive & 79 & 5110 & 11 & 617 & 1.74 & 158 & 10220 & 22 & 1586 & 3.67 \\
\hline & MSNmax & 59 & 7764 & 7 & 779 & 1.53 & 130 & 15890 & 14 & 2148 & 3.50 \\
\hline \multirow[t]{2}{*}{ pltexpA4 } & Naive & 106 & 2257 & 9 & 146 & 1.25 & 212 & 4514 & 20 & 225 & 2.46 \\
\hline & MSNmax & 60 & 2543 & 8 & 256 & 0.93 & 120 & 5410 & 14 & 314 & 2.08 \\
\hline \multirow[t]{2}{*}{ world } & Naive & 79 & 5289 & 9 & 667 & 1.89 & 158 & 10578 & 19 & 2204 & 3.73 \\
\hline & MSNmax & 65 & 8316 & 7 & 836 & 1.66 & 134 & 13638 & 16 & 2442 & 3.38 \\
\hline \multicolumn{12}{|c|}{ Normalized averages over Naive } \\
\hline & Naive & 1.00 & 1.00 & 1.00 & 1.00 & 1.00 & 1.00 & 1.00 & 1.00 & 1.00 & 1.00 \\
\hline & MSNmax & 0.55 & 1.33 & 0.79 & 2.10 & 0.78 & 0.56 & 1.37 & 0.65 & 1.52 & 0.82 \\
\hline
\end{tabular}

TABLE 5.5

24-way partitioning and sequential matrix-vector multiply times in msecs.

\begin{tabular}{l|ll|lr|c}
\hline \multirow{2}{*}{ Matrix } & \multicolumn{3}{|c|}{ Partitioning times } & \multirow{2}{*}{$\begin{array}{c}\text { Seq. } \\
\end{array}$} & \multicolumn{2}{|c|}{ Phase 1 } & \multicolumn{2}{c}{ Phase 2 } \\
& Method & Time & Method & Time & time \\
\hline lhr17 & PaToH & 6100 & Naive & 32 & 19.56 \\
& & & PaToH-fix & 13084 & \\
& & & MSN & 3988 & \\
& & & MSNmax & 3885 & \\
\hline pig-very & PaToH & 20960 & Naive & 12 & 30.37 \\
& & & PaToH-fix & 2281 & \\
& & & MSN & 1086 & \\
& & & MSNmax & 1022 & \\
\hline fxm4-6 & PaToH & 2950 & Naive & 2 & 13.19 \\
& & & PaToH-fix & 58 & \\
& & & MSN & 112 & \\
& & & MSNmax & 81 & \\
\hline
\end{tabular}

Ihr17 with $\mathrm{PaToH}$-fix. As expected, the communication hypergraphs are smaller than the respective computational hypergraphs. However, some communication hypergraphs might have very large net sizes because of the small number of nets. Matrix lhr17 is an example of such a case with the large average net size of $n n z(C) / K=1225$ in the communication hypergraph versus the small average net size of $n n z(A) / N=22$ 
in the computational hypergraph. This explains the above exceptional experimental outcome because running times of matching heuristics, used in the coarsening step of PaToH, increase with the sum of squares of net sizes [8] (see also Theorem 5.5 in [21]).

Comparing the running times of communication-hypergraph partitioning methods, Naive takes an insignificant amount of time as seen in Table 5.5. Direct $K$-way partitioning approaches are expected to be faster than the recursive-bisection based $\mathrm{PaToH}$-fix because of the single coarsening step as compared with $K-1=23$ coarsening steps. As expected, $M S N$ and $M S N$ max take considerably less time than $\mathrm{PaToH}-\mathrm{fix}$ except in partitioning communication-hypergraph of $\mathrm{fxm} 4-6$, which has a moderate average net size. As seen in the table, the second-phase methods MSN and MSNmax introduce much less preprocessing overhead than the first phase. The partitionings obtained by MSNmax for $1 \mathrm{hr} 17$, pig-very, and fxm4-6 matrices lead to speedup values of $8.89,11.1$, and 13.9, respectively, in row-parallel matrix-vector multiplies on our 24-processor PC cluster.

6. Conclusion. We proposed a two-phase approach that encapsulates multiple communication-cost metrics in 1D partitioning of structurally unsymmetric square and rectangular sparse matrices. The objective of the first phase was to minimize the total message volume and maintain computational-load balance within the framework of the existing 1D matrix partitioning methods. For the second phase, communicationhypergraph models were proposed. Then, the problem of minimizing the total message latency while maintaining the balance on message-volume loads of processors was formulated as a hypergraph partitioning problem on communication hypergraphs. Several methods were proposed for partitioning communication hypergraphs. One of these methods was tailored to encapsulate the minimization of the maximum message count per processor. We tested the performance of the proposed models and the associated partitioning methods on a wide range of large unsymmetric square and rectangular sparse matrices. In these experiments, the proposed two-phase approach achieved substantial improvements in terms of communication-cost performance metrics. We also implemented parallel matrix-vector and matrix-matrix-transpose-vector multiplies using MPI to see whether the theoretical improvements achieved in the given performance metrics hold in practice. Experiments on a PC cluster showed that the proposed approach can achieve substantial improvements in parallel run times.

Parallel matrix-vector multiply $y=A x$ is one of the basic parallel reduction algorithms. Here, the $x$-vector entries are the input, and the $y$-vector entries are the output of the reduction. The matrix $A$ corresponds to the mapping from the input to the output vector entries. Çatalyürek and Aykanat [10] briefly list several practical problems that involve this correspondence. Hence, the proposed two-phase approach can also be used in reducing the communication overhead in such practical reduction problems.

Acknowledgment. The authors thank the anonymous referees, whose suggestions greatly improved the presentation of the paper.

\section{REFERENCES}

[1] C. J. Alpert, A. E. Caldwell, A. B. Kahng, and I. L. Markov, Hypergraph partitioning with fixed vertices, IEEE Trans. Computer-Aided Design, 19 (2000), pp. 267-272.

[2] C. Aykanat, A. Pinar, and U. V. Çatalyürek, Permuting sparse rectangular matrices into block-diagonal form, SIAM J. Sci. Comput., 25 (2004), pp. 1860-1879. 
[3] M. BenzI, Preconditioning techniques for large linear systems: A survey, J. Comput. Phys., 182 (2002), pp. 418-477.

[4] M. Benzi And M. Tuma, A comparative study of sparse approximate inverse preconditioners, Appl. Numer. Math., 30 (1999), pp. 305-340.

[5] G. Burns, R. Daoud, And J. Vaigl, LAM: An open cluster environment for MPI, in Proceedings of Supercomputing Symposium '94, J. W. Ross, ed., University of Toronto, Ontario, Canada, 1994, pp. 379-386.

[6] U. V. Çatalyürek, Hypergraph Models for Sparse Matrix Partitioning and Reordering, Ph.D. thesis, Computer Engineering and Information Science, Bilkent University, Ankara, Turkey, 1999.

[7] U. V. Çatalyürek and C. Aykanat, Decomposing irregularly sparse matrices for parallel matrix-vector multiplications, in Parallel Algorithms for Irregularly Structured Problems, Lecture Notes in Comput. Sci. 1117, Springer-Verlag, Berlin, 1996, pp. 75-86.

[8] U. V. Çatalyürek and C. AyKanat, Hypergraph-partitioning based decomposition for parallel sparse-matrix vector multiplication, IEEE Trans. Parallel and Distributed Systems, 10 (1999), pp. 673-693.

[9] U. V. Çatalyürek and C. Aykanat, PaToH: A Multilevel Hypergraph Partitioning Tool, Version 3.0, Tech. rep. BU-CE-9915, Computer Engineering Department, Bilkent University, Ankara, Turkey, 1999.

[10] U. V. ÇAtalyürek and C. Aykanat, A hypergraph-partitioning approach for coarse-grain decomposition, in Proceedings of the IEEE Symposium on Scientific Computing, Denver, CO, 2001, pp. 10-16.

[11] A. DaşDan And C. AYkanat, Two novel multiway circuit partitioning algorithms using relaxed locking, IEEE Trans. Computer-Aided Design of Integrated Circuits and Systems, 16 (1997), pp. 169-178.

[12] J. J. Dongarra and T. H. Dunigan, Message-passing performance of various computers, Concurrency-Practice and Experience, 9 (1997), pp. 915-926.

[13] C. M. Fiduccia and R. M. Mattheyses, A linear-time heuristic for improving network partitions, in Proceedings of the 19th ACM/IEEE Design Automation Conference, ACM, New York, IEEE Computer Society, Los Alamitos, CA, 1982, pp. 175-181.

[14] R. W. Freund And N. M. Nachtigal, QMR: A quasi-minimal residual algorithm for nonHermitian linear systems, Numer. Math., 60 (1991), pp. 315-339.

[15] G. H. Golub and C. F. V. Loan, Matrix Computations, 3rd ed., The Johns Hopkins University Press, Baltimore, 1996.

[16] M. J. Grote And T. HuCkLe, Parallel preconditioning with sparse approximate inverses, SIAM J. Sci. Comput., 18 (1997), pp. 838-853.

[17] A. Gupta, Watson Graph Partitioning Package, Tech. rep. RC 20453, IBM T. J. Watson Research Center, Yorktown Heights, NY, 1996.

[18] M. Hegland, Description and Use of Animal Breeding Data for Large Least Squares Problems, Tech. rep. TR-PA-93-50, CERFACS, Toulouse, France, 1993.

[19] B. Hendrickson, Graph partitioning and parallel solvers: Has the emperor no clothes?, in Solving Irregularly Structured Problems in Parallel, Lecture Notes in Comput. Sci. 1457, Springer-Verlag, Berlin, 1998, pp. 218-225.

[20] B. Hendrickson And T. G. Kolda, Graph partitioning models for parallel computing, Parallel Comput., 26 (2000), pp. 1519-1534.

[21] B. Hendrickson and T. G. Kolda, Partitioning rectangular and structurally unsymmetric sparse matrices for parallel processing, SIAM J. Sci. Comput., 21 (2000), pp. 20482072.

[22] B. Hendrickson and R. Leland, The Chaco Users's Guide, Version 2.0, Tech. rep. SAND952344, Sandia National Laboratories, Albuquerque, NM, 1995.

[23] E. Horowitz And S. SAhni, Fundamentals of Computer Algorithms, Computer Science Press, Rockville, MD, 1978.

[24] G. Karypis AND V. Kumar, MeTiS: A Software Package for Partitioning Unstructured Graphs, Partitioning Meshes, and Computing Fill-Reducing Orderings of Sparse Matrices Version 3.0, Tech. rep., Department of Computer Science and Engineering, Army HPC Research Center, University of Minnesota, Minneapolis, MN, 1998.

[25] G. Karypis and V. Kumar, Multilevel Algorithms for Multi-constraint Graph Partitioning, Tech. rep. 98-019, Department of Computer Science, Army HPC Research Center, University of Minnesota, Minneapolis, MN, 1998.

[26] G. Karypis, V. Kumar, R. Aggarwal, and S. Shekhar, hMeTiS: A Hypergraph Partitioning Package Version 1.0.1, Department of Computer Science and Engineering, Army HPC Research Center, University of Minnesota, Minneapolis, MN, 1998. 
[27] B. W. Kernighan and S. Lin, An efficient heuristic procedure for partitioning graphs, The Bell System Technical Journal, 49 (1970), pp. 291-307.

[28] T. Lengauer, Combinatorial Algorithms for Integrated Circuit Layout, Wiley-Teubner, Chichester, UK, 1990.

[29] H. Özaktaş, Algorithms for Linear and Convex Feasibility Problems: A Brief Study of Iterative Projection, Localization and Subgradient Methods, Ph.D. thesis, Industrial Engineering Department, Bilkent University, Ankara, Turkey, 1998.

[30] H. Özaktas, M. AKGüL, ANd M. Ç. PinaR, A parallel surrogate constraint approach to the linear feasibility problem, in Applied Parallel Computing: Computations in Physics, Chemistry, and Engineering Science, Lecture Notes in Comput. Sci. 1184, Springer-Verlag, Berlin, 1996, pp. 565-574.

[31] C. C. Paige And M. A. SAunders, LSQR: An algorithm for sparse linear equations and sparse least squares, ACM Trans. Math. Software, 8 (1982), pp. 43-71.

[32] A. Pinar, U. V. Çatalyürek, C. Aykanat, and M. Ç. Pinar, Decomposing linear programs for parallel solution, in Proceedings of the 2nd International Workshop on Parallel Computing, Lecture Notes in Comput. Sci. 1041, Springer-Verlag, Berlin, 1996, pp. 473-482.

[33] A. Pinar and B. Hendrickson, Graph partitioning for complex objectives, in Proceedings of Irregular 2001 Eighth International Workshop on Solving Irregularly Structured Problems in Parallel, San Francisco, CA, 2001.

[34] Y. SAAD, Iterative Methods for Sparse Linear Systems, PWS Publishing, Boston, 1996.

[35] L. A. SANChIS, Multiple-way network partitioning, IEEE Trans. Comput., 38 (1989), pp. 62-81.

[36] L. A. SAnchis, Multiple-way network partitioning with different cost functions, IEEE Trans. Comput., 42 (1993), pp. 1500-1504.

[37] K. Schloegel, G. Karypis, and V. Kumar, A New Algorithm for Multi-Objective Graph Partitioning, Tech. rep. 99-003, Department of Computer Science, Army HPC Research Center, University of Minnesota, Minneapolis, MN, 1999.

[38] T. Sterling, D. Savarese, D. J. Becker, J. E. Dorband, U. A. Ranaweke, and C. V. PACKER, BEOWULF: A parallel workstation for scientific computation, in Proceedings of the 24th International Conference on Parallel Processing, Oconomowoc, WI, 1995, pp. $11-34$.

[39] E. Turna, Parallel Algorithms for the Solution of Large Sparse Inequality Systems on Distributed Memory Architectures, Master's thesis, Computer Engineering and Information Science, Bilkent University, Ankara, Turkey, 1998.

[40] K. Yang And K. G. Murty, New iterative methods for linear inequalities, J. Optim. Theory Appl., 72 (1992), pp. 163-185. 